\title{
A human brainstem glioma xenograft model enabled for bioluminescence imaging
}

\author{
Rintaro Hashizume $\cdot$ Tomoko Ozawa $\cdot$ Eduard B. Dinca $\cdot$ \\ Anuradha Banerjee $\cdot$ Michael D. Prados · Charles D. James · \\ Nalin Gupta
}

Received: 10 March 2009/Accepted: 22 June 2009/Published online: 8 July 2009

(c) The Author(s) 2009. This article is published with open access at Springerlink.com

\begin{abstract}
Despite the use of radiation and chemotherapy, the prognosis for children with diffuse brainstem gliomas is extremely poor. There is a need for relevant brainstem tumor models that can be used to test new therapeutic agents and delivery systems in pre-clinical studies. We report the development of a brainstem-tumor model in rats and the application of bioluminescence imaging (BLI) for monitoring tumor growth and response to therapy as part of this model. Luciferase-modified human glioblastoma cells from five different tumor cell sources (either cell lines or serially-passaged xenografts) were implanted into the pontine tegmentum of athymic rats using an implantable guide-screw system. Tumor growth was monitored by BLI and tumor volume was calculated by three-dimensional measurements from serial histopathologic sections. To evaluate if this model would allow detection of therapeutic response, rats bearing brainstem U-87 MG or GS2 glioblastoma xenografts were treated with the DNA methylating agent temozolomide (TMZ). For each of the tumor cell sources tested, BLI monitoring revealed progressive tumor growth in all animals, and symptoms caused by tumor burden were evident 26-29 days after implantation of U-87 MG, U-251 MG, GBM6, and GBM14 cells, and
\end{abstract}

R. Hashizume - T. Ozawa - E. B. Dinca ·

M. D. Prados - C. D. James · N. Gupta $(\bowtie)$

Department of Neurological Surgery, Brain Tumor Research

Center, University of California, 505 Parnassus Ave.,

Room M779, San Francisco, CA 94143-0112, USA

e-mail: GuptaN@neurosurg.ucsf.edu

A. Banerjee

Division of Pediatric Oncology, Department of Pediatrics,

University of California, San Francisco, CA, USA
37-47 days after implantation of GS2 cells. Histopathologic analysis revealed tumor growth within the pons in all rats and BLI correlated quantitatively with tumor volume. Variable infiltration was evident among the different tumors, with GS2 tumor cells exhibiting the greatest degree of infiltration. TMZ treatment groups were included for experiments involving U-87 MG and GS2 cells, and in each case TMZ delayed tumor growth, as indicated by BLI monitoring, and significantly extended survival of animal subjects. Our results demonstrate the development of a brainstem tumor model in athymic rats, in which tumor growth and response to therapy can be accurately monitored by BLI. This model is well suited for pre-clinical testing of therapeutics that are being considered for treatment of patients with brainstem tumors.

Keywords Brainstem tumor - Animal model . Bioluminescence $\cdot$ Temozolomide

\section{Introduction}

Brainstem gliomas represent $10-15 \%$ of brain tumors in children and show varying degrees of malignancy [1]. The majority of brainstem gliomas are diffuse pontine tumors and patients with these tumors rarely survive beyond 2 years from initial diagnosis [1]. Factors that contribute to the dismal prognosis associated with diffuse pontine gliomas include infiltrative nature and anatomic location in an eloquent area of the brain which precludes surgical resection [1, 2]. Although many radiation and chemotherapy regimens have been used to treat diffuse pontine gliomas, the long-term outcome has not changed substantially [1,2].

Recent advances in the treatment of high-grade supratentorial gliomas in adults have led to the development of 
more potent and specific anticancer agents and new drug delivery systems [2-6]. Undoubtedly, the translation of these new therapies and approaches to the treatment of diffuse pontine gliomas would be facilitated through use of an animal model designed specifically for preclinical therapeutic testing. The model that best mimic the human clinical condition involves transplantation of human glioma cells that have been well-characterized in their response to chemotherapeutic agents and ionizing radiation [7-10].

When using intracranial xenograft models, therapeutic efficacy is usually measured by the time required for animals to develop symptoms associated with tumor burden. An alternative approach for assessing intracranial xenograft response to therapy is bioluminescence imaging (BLI), which detects photon emissions associate with an ATP-dependent process catalyzed by metabolically active cells that express a luciferase reporter gene. BLI has been shown to allow noninvasive, quantitative, and real-time monitoring of tumor growth and response to therapy in many small-animal models [11-16].

Here, we report the development and application of an orthotopic brainstem tumor model in athymic rats using a guide-screw system to implant human glioblastoma cells, and that is followed by BLI monitoring. In association with this model system, BLI accurately reflects orthotopic tumor growth and is able to measure tumor response to the DNA methylating agent temozolomide (TMZ). Our model system with adaptation for BLI should prove useful for preclinical testing of therapeutics for treating patients with brainstem tumors.

\section{Materials and methods}

\section{Cell culture}

U-87 MG and U-251 MG human glioblastoma cells were obtained from the Department of Neurological Surgery Tissue Bank at the University of California, San Francisco (UCSF), and were propagated as exponentially growing monolayers in complete medium consisting of Eagle's minimal essential medium supplemented with $10 \%$ fetal bovine serum and non-essential amino acids. The GS2 cell line was obtained from Manfred Westphal, Department of Neurological Surgery, University Hospital Eppendorf, Hamburg, Germany, and maintained as a neurosphere culture, as previously described [17]. GBM6 and GBM14 cells were obtained from C. David James, Department of Neurological Surgery, UCSF, and propagated as subcutaneous xenografts that were harvested and prepared for intracranial injection, as previously described [18].
Modification of tumor cells with firefly-luciferaseexpressing reporter

U-87 MG, U-251 MG, GBM6, GBM14, and GS2 cells were transduced with a lentiviral vector containing firefly luciferase (Fluc) under the control of the spleen focus forming virus (SFFV) promoter. Lentiviral vectors were generated by transfection of 293T (human embryonal kidney) cells with plasmids encoding the vesicular stomatitis virus $\mathrm{G}$ envelope, gag-pol, and Fluc genes [16, 19]. Viral vectors were harvested from supernatant 48 hours after transfection, filtered, and then used to infect glioblastoma cells. Cells were screened for transfection efficiency by treatment with luciferin (D-luciferin potassium salt, $150 \mathrm{mg} / \mathrm{kg}$, Gold Biotechnology, St Louis, MO) in vitro and examination by a Xenogen IVIS Lumina System (Xenogen Corp., Alameda, CA). More than $80 \%$ of cells were transfected.

Animals

Six-week-old male athymic rats (rnu/rnu, homozygous) were purchased from the National Cancer Institute (Frederick, MD). Rats were housed in an animal facility and were maintained in a temperature-controlled and lightcontrolled environment with an alternating 12-hour light/ dark cycle. All protocols were approved by the UCSF Institutional Animal Care and Use Committee.

Surgical procedure for implantation of tumor cells

Before injecting tumor cells into the brainstem, rats were anesthetized by intraperitoneal injection of $75 \mathrm{mg} / \mathrm{kg}$ of ketamine and $7.5 \mathrm{mg} / \mathrm{kg}$ of xylazine. Anesthetized rats were then positioned in a stereotactic device (David Kopf Instruments, Tujunga, CA) using ear bars. A burr hole was drilled through the skull $1.0 \mathrm{~mm}$ behind the lambda, and $9.6 \mathrm{~mm}$ deep from the inner surface of the skull. $1 \times 10^{5}$ tumor cells suspended in $1 \mu \mathrm{l}$ HBSS were injected slowly (over 1 minute) into the pontine tegmentum using a guidescrew system. All procedures were carried out under sterile conditions.

\section{In vivo BLI monitoring}

In vivo BLI was performed with the Xenogen IVIS Lumina System coupled LivingImage software for data acquisition (Xenogen Corp.). Rats were anesthetized with $75 \mathrm{mg} / \mathrm{kg}$ of ketamine and $7.5 \mathrm{mg} / \mathrm{kg}$ of xylazine and imaged $12 \mathrm{~min}$ after intraperitoneal injection of luciferin. Signal intensity was quantified within a region of interest over the head that was defined by the LivingImage software. There was considerable variation in luminescence among tumor- 
Fig. 1 Comparison of tumor morphology associated with different GBM cell lines following implantation into the rodent pons. a $1 \times 10^{5}$ luciferase-modified U-87 MG, U-251 MG, GBM6, GBM 14, and GS2 glioblastoma cells were injected into brainstem in athymic rats using an implantable guide-screw system. b GS2 cells showed the greatest degree of infiltration within the pons, but show a lesser extent of infiltration than is evident following supratentorial injection in athymic mice
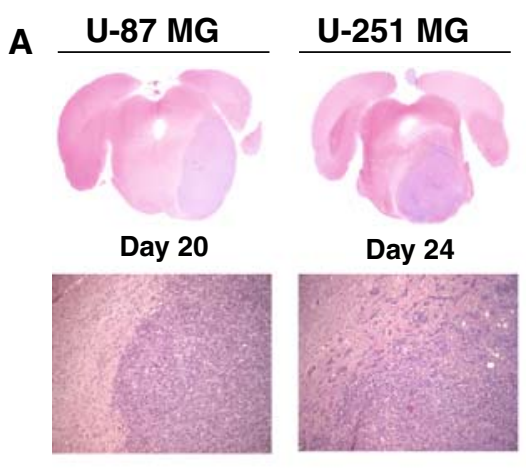

Day 24
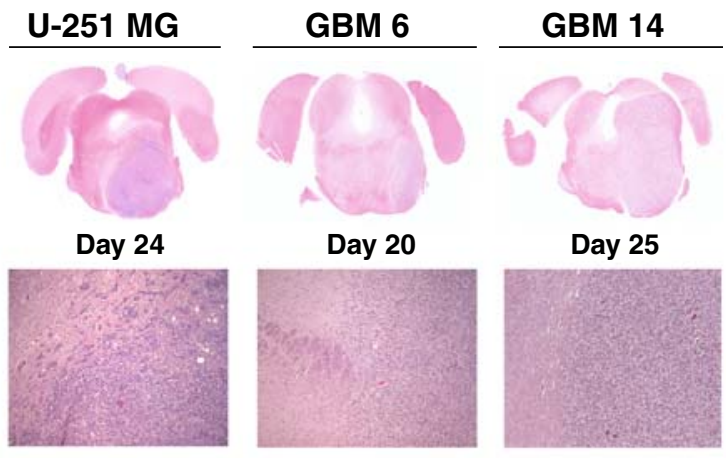

Day 25

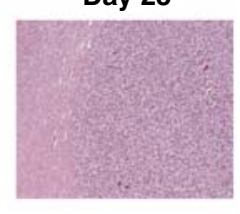

GS2

B

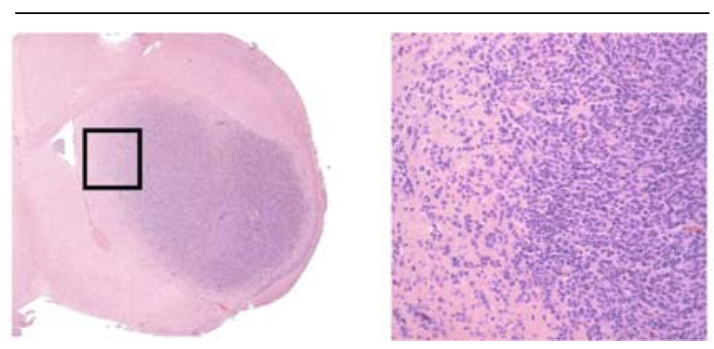

bearing mice. To facilitate comparison of growth rates, each mouse's luminescence readings were normalized against its own luminescence reading at day 14 (last BLI before initiation of therapy), thereby allowing each mouse to serve as its own control [14].

Growth kinetics of brainstem tumor xenografts

Eight rats were injected with $1 \times 10^{5}$ luciferase-modified U-87 MG cells into the brainstem and intratumoral luciferase activity was monitored by BLI. BLI was performed twice a week beginning 3 days after tumor cells implantation. Two rats each were euthanized at days 10 , 15,20 and 25 following implantation and immediately after BLI. Brains were fixed in $10 \%$ buffered formalin and embedded in paraffin, sectioned at $250 \mu \mathrm{m}$ intervals, and then stained with hematoxillin-eosin (H\&E). For the volumetric assessment of intracranial tumor, tumor regions were identified on serially-sectioned H\&E stained slides and digital images of these slides were acquired and organized as volume wrapper files, in which images were in spatial registration. Analyze 7.0 software (AnalyzeDirect, Inc., Overland Park, KS) was used to determine three-dimensional tumor volumes for each set of spatially registered images as a function of pixel dimensions. Estimated relative volumes were compared with corresponding optical imaging measurements to determine the extent of correlation between the two measurements.
Treatment of brainstem tumors in athymic rats with TMZ

To evaluate the therapeutic response of brainstem tumor xenografts, rats receiving brainstem injections of luciferase-modified U-87 MG or GS2 cells were randomly assigned to vehicle control (Ora-Plus, Paddock Laboratories, Inc., North Minneapolis, MN) or TMZ treatment (Temodar, Schering corp., Kenilworth, NJ) groups. For U-87 MG brainstem xenografts, the treatment group received daily dose of $50 \mathrm{mg} / \mathrm{kg}$ of TMZ by oral gavage for 5 consecutive. Treatment was initiated on day 15 when brainstem tumors had achieved a log growth phase, as indicated by BLI monitoring. For GS2 brainstem xenografts, the treatment group received a single dose of $100 \mathrm{mg} / \mathrm{kg}$ TMZ by oral gavage on day 28 . All rats were monitored every day for the development of symptoms related to tumor growth and twice weekly by BLI. Mean BLI values for control and treatment groups were calculated and plotted according to the corresponding day of imaging. Rats were euthanized when they exhibited symptoms indicative of significant compromise to neurological function.

\section{TUNEL staining}

To determine if TMZ caused an apoptotic response in tumor cells, the brains from one control and one TMZtreated rat were resected immediately following completion of the treatment regimen at day 5 , placed in formalin 
Fig. 2 Association between bioluminescence signal and corresponding tumor volume in rats receiving brainstem injection of U-87 MG cells. a Eight rats were injected with luciferase-modified U-87 MG glioblastoma cells and bioluminescence monitoring was performed twice a week, beginning 3 days after tumor cell implantation. Two rats each were euthanized on days 10,15 , 20 , and 25 following BLI.

Histopathologic analysis reveals progressive U-87 MG tumor growth from day 10 to 25 (arrows, $2 \times$ magnification). b Bioluminescence imaging (BLI) shows a corresponding signal increase from the tumors. c Brains from rats euthanized at days $10,15,20$ and 25 after U-87 MG tumor cells implantation were serially sectioned at $250 \mu \mathrm{m}$ intervals.

Tumor size from digital images of serially-registered sections were compared with corresponding BLI measurements to determine the extent of correlation.

Quantitative comparison of relative tumor volume with corresponding luminescence values $\left(R^{2}=0.9467\right)$
A U-87 MG

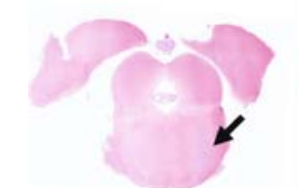

Day 10

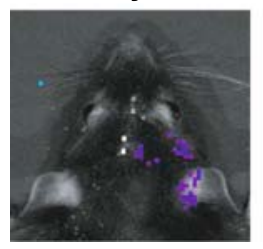

Day 15
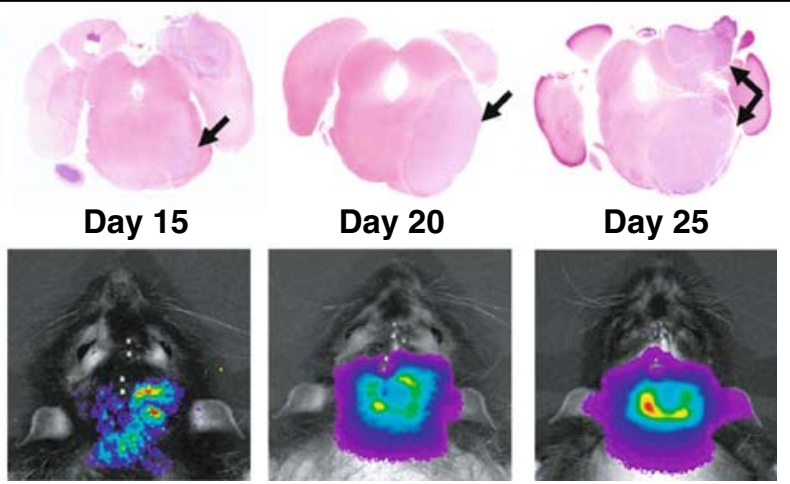

Day 20

Day 25
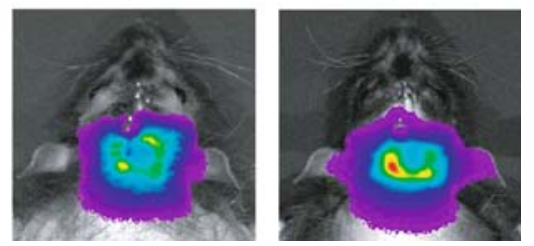

B

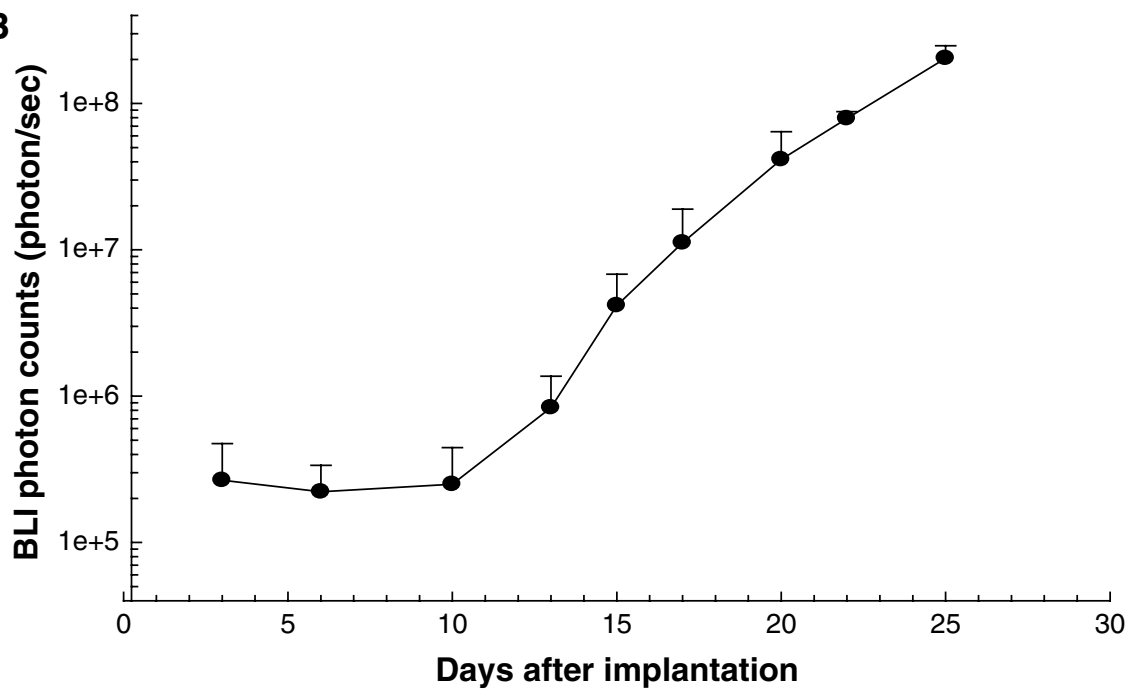

C

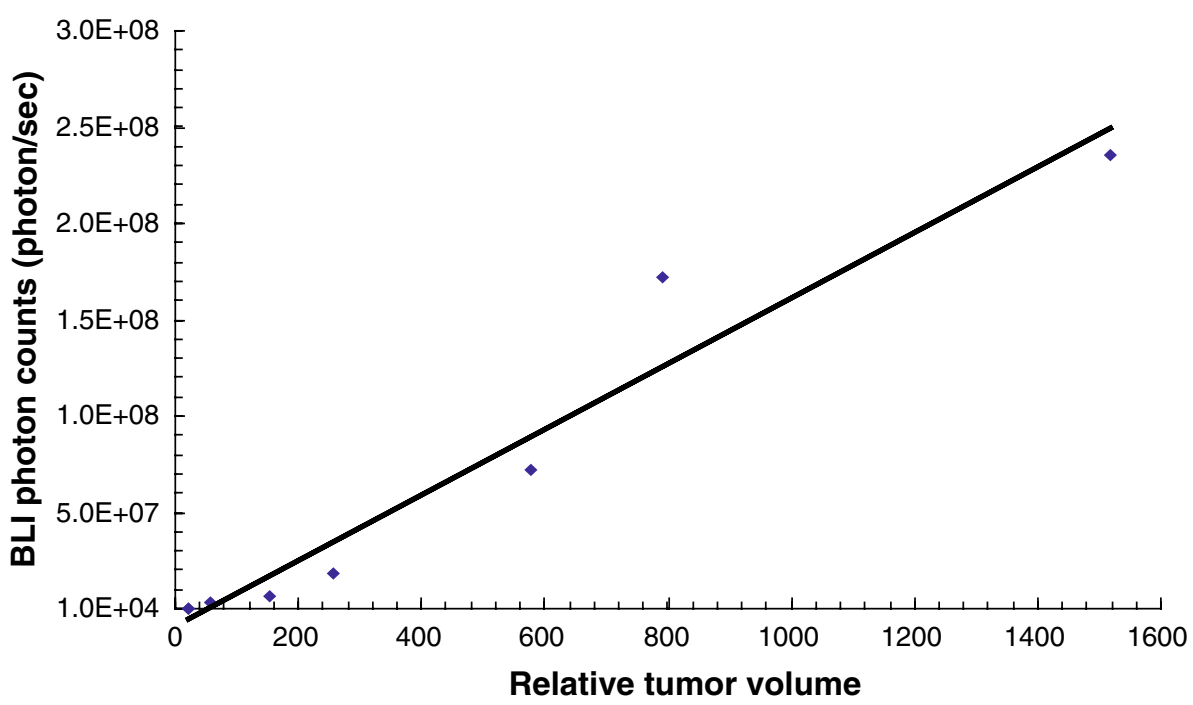

and embedded in paraffin. The paraffin-embedded sections were assessed for apoptosis using terminal nucleotidyl transferase-mediated nick end labeling (TUNEL) staining. TUNEL staining was performed using the ApopTag
Peroxidase In situ Apoptosis detection kit (CHEMICON, Temecula, CA), according to the manufacturer's instruction. Counterstaining was performed using hematoxillin. The number of TUNEL-positive cells was counted in four 
high-power fields $(200 \times$ magnification $)$ from each rat, and mean values determined.

\section{Statistical analysis}

The Kaplan-Meier estimator was used to generate survival curves, and differences between survival curves were calculated using a log-rank test.

\section{Results}

Progressive growth of brainstem xenografts measured by BLI

Injection of luciferase-modified U-87 MG, U-251 MG, GBM6, GBM14, and GS2 human glioblastoma cells into athymic rats resulted in $100 \%$ tumor take (Figs. 1, 2). No animals suffered adverse effects from the direct brainstem injection procedure. All animals developed typical posterior fossa symptoms, such as torticollis and nystagmus. Survival of rats, as determined by criteria necessitating euthanasia, ranged from 26 to 29 days after implantation of U-87 MG, U-251 MG, GBM6, and GBM14 cells, and 37 to 47 days after implantation of GS2 cells. Histopathologic analysis revealed large tumors within the pons in all rats (Fig. 1a) and corresponding BLI revealed readily detectable and quantifiable signal from the tumors. Although most of the cell lines showed a relatively well-circumscribed pattern of growth, GS2 cells infiltrated more widely through the pons with a pattern that more closely resembles the human disease (Fig. 1b, Table 1).

In a time course study involving tumor monitoring by BLI, rats were injected with luciferase-modified U-87 MG cells and euthanized at selected time points following implantation. Small tumors were detected on day 10 with increasing tumor size up to day 25 . On day 25 , solid tumor was evident in the right pons with extension over the brain surface (Fig. 2a). BLI revealed increasing signal intensity corresponding to increasing tumor volume in all animals (Fig. 2a, b). Implanted tumor cells achieved a logarithmic growth subsequent to day 10 following implantation, at which time injected rodents were asymptomatic.

Correlation between bioluminescence and corresponding tumor volume

H\&E stained sections of each tumor bearing brain, from the time course study, were serially registered and used to estimate tumor volume. Tumor volumes were compared with corresponding luminescence signals obtained from rats immediately prior to euthanasia. There was significant correlation between tumor volume and luminescence signaling $\left(R^{2}=0.9467\right.$; Fig. 2c).

Brainstem tumor response to $\mathrm{TMZ}$ treatment

To evaluate the therapeutic response of brainstem tumor xenografts, we used BLI monitoring to measure tumor response to TMZ in both $\mathrm{U}-87 \mathrm{MG}$ and GS2 cells. The normalized luminescence plot for the control group showed progressively increasing luminescence until rats developed neurological symptoms indicative of a moribund condition (Figs. 3, 4). In comparison to the control group rodents with U-87 MG tumors, luminescence in rats receiving TMZ treatment was substantially reduced at day 19 , when the treatment was completed, and remained low until day 42 following implantation (Fig. 3b). Survival analysis revealed that the median survival of rats treated with TMZ was significantly extended relative to controls (53.5 days vs. 27 days, $P=0.0033$; Fig. 3a). To evaluate whether TMZ caused apoptosis of U-87 MG tumor cells, TUNEL staining was used to examine tumor tissue from one control and one treated rat that were sacrificed at the completion of TMZ treatment. Increased numbers of TUNEL-positive cells were present in the U-87 MG tumor following TMZ treatment (mean value $=26 \mathrm{TMZ}$ treated vs. 0.75 control, Fig. 3c).

In rodents with GS2 tumors, TMZ treatment resulted in complete loss of detectable luminescence (Fig. 4a), and TMZ treated animals were sacrificed at day 72 (Fig. 4b), with subsequent histopathologic analysis yielding no indication of tumor in the brains of treated mice (data not

Table 1 Description of glioblastoma cell lines used to establish brainstem tumors

\begin{tabular}{llll}
\hline Cell line & Propagation method & Growth pattern & Median survival (days) \\
\hline U-87 MG & Monolayer cell culture with serum & Well-circumscribed & 28 \\
U-251 MG & Monolayer cell culture with serum & Infiltrative & 26 \\
GBM6 & Subcutaneous tumor in mouse & Infiltrative & 29 \\
GBM14 & Subcutaneous tumor in mouse & Circumscribed & 28 \\
GS2 & Neurosphere cell culture with EGF, FGF, N2, B27 & Extensive invasion at tumor margin & 44 \\
\hline
\end{tabular}


Fig. 3 Bioluminescence monitoring of U-87 MG brainstem tumor xenografts treated with TMZ. a U-87 MG tumor bearing rats were treated with a daily dose of $50 \mathrm{mg} / \mathrm{kg}$ of TMZ or control vehicle delivered by oral gavage for 5 consecutive days beginning on day 15. Bioluminescence measurements for each rat were normalized against

corresponding readings obtained at the beginning of therapy.

TMZ treatment group $(n=4$, white circle) shows a decreased normalized luminescence value through day 42 , in contrast to the increasing luminescence value of control group, that was first evident at day 19 imaging $(n=5$, black circle $)$. b TMZ treatment significantly prolonged median survival $(P=0.0033)$ from 27 days in the control group $(n=5$, black circle) to 53.5 days in the TMZ treatment group ( $n=4$, white circle). c TUNEL staining reveals an increase in the number of TUNEL-positive cells in tumor from the rat treated with TMZ, as compared with the tumor from the control animal (100× magnification; mean value $=26$ vs. 0.75 )
A

U-87 MG

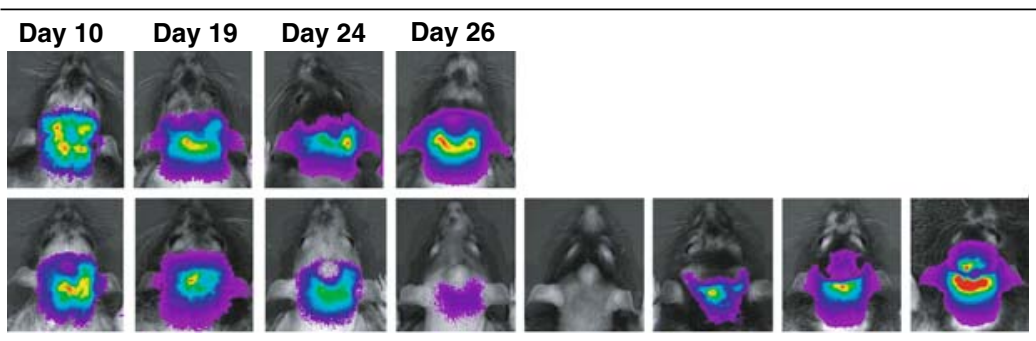

$\begin{array}{llllllll}\text { Day } 10 & \text { Day } 19 & \text { Day } 24 & \text { Day } 29 & \text { Day } 34 & \text { Day } 42 & \text { Day } 50 & \text { Day } 42\end{array}$

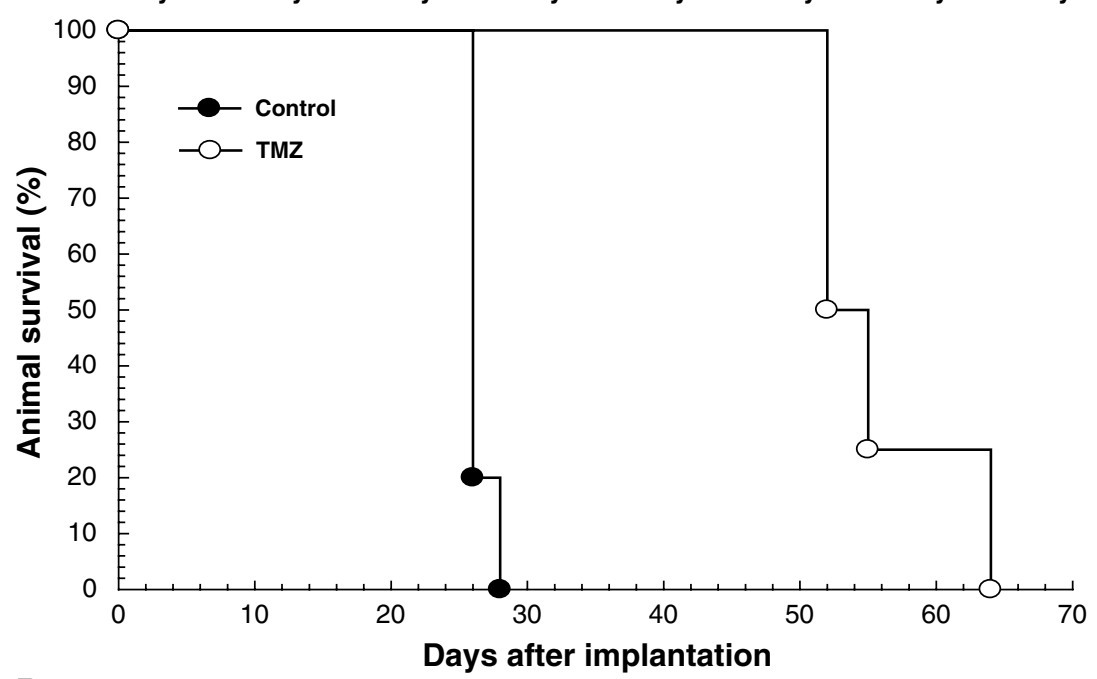

B

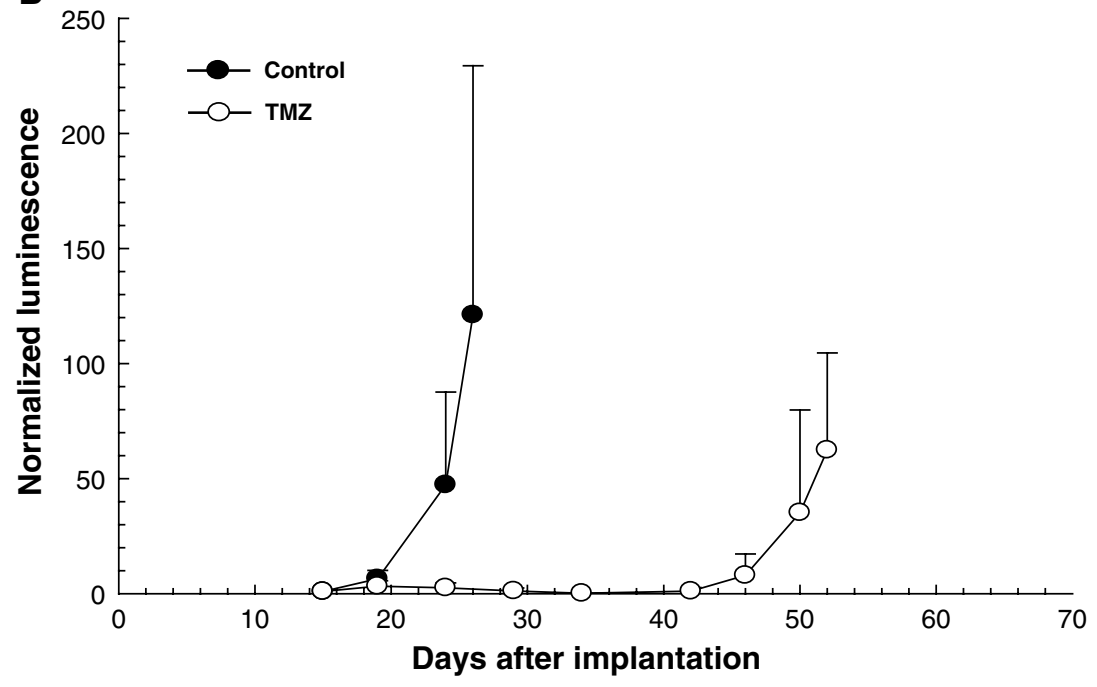

C
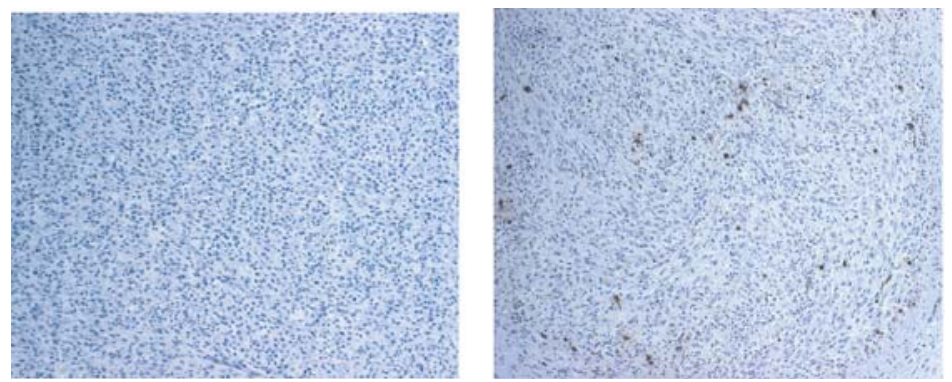
Fig. 4 Bioluminescence monitoring of GS2 brainstem tumor xenografts treated with TMZ. a GS2 tumor bearing rats were treated with a single dose of $100 \mathrm{mg} / \mathrm{kg}$ of TMZ or control vehicle delivered by oral gavage on day 28. Bioluminescence measurements for each rat were normalized against corresponding readings obtained at the beginning of therapy.

TMZ treatment group $(n=4$, white circle) shows decreasing normalized luminescence until no signal is evident in treated mice, whereas control group mice show steadily increased luminescence from day 26 onward ( $n=5$, black circle). b TMZ treatment significantly prolonged median survival $(P<0.0001)$ from 44 days in the control group ( $n=4$, black circle) to $>72$ days in the TMZ treatment group ( $n=4$, white circle), at which time all TMZ treatment group rats were sacrificed and determined as being devoid of viable tumor
A

GS2

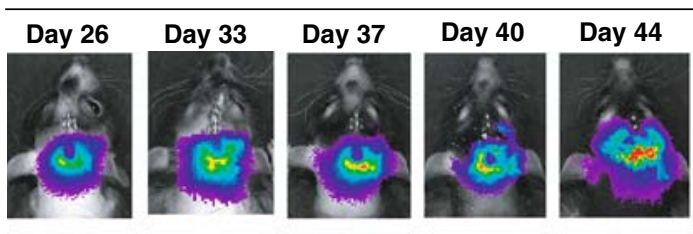

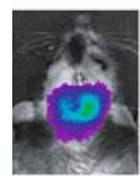

Day 26

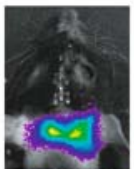

Day 33

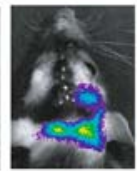

Day 37

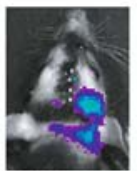

Day 40

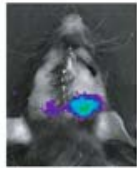

Day 44

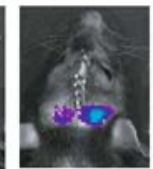

Day 48

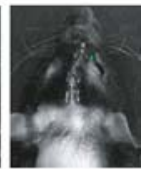

Day 55

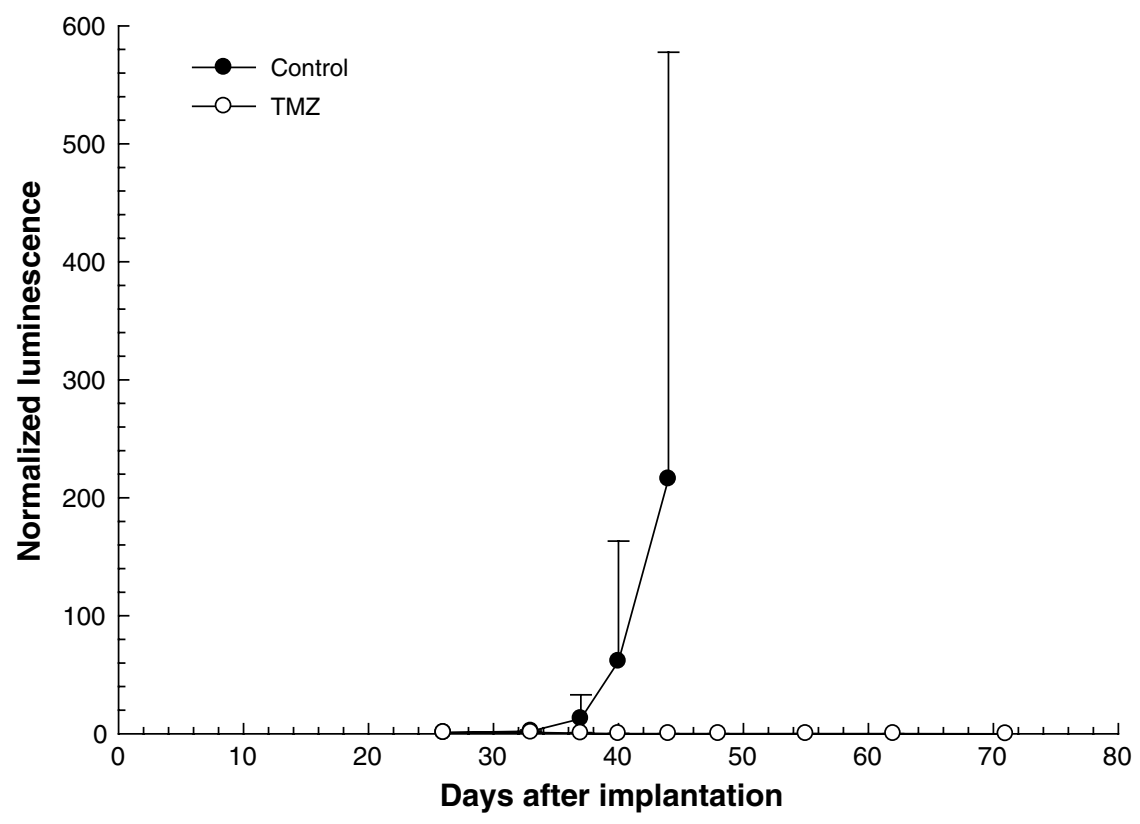

B

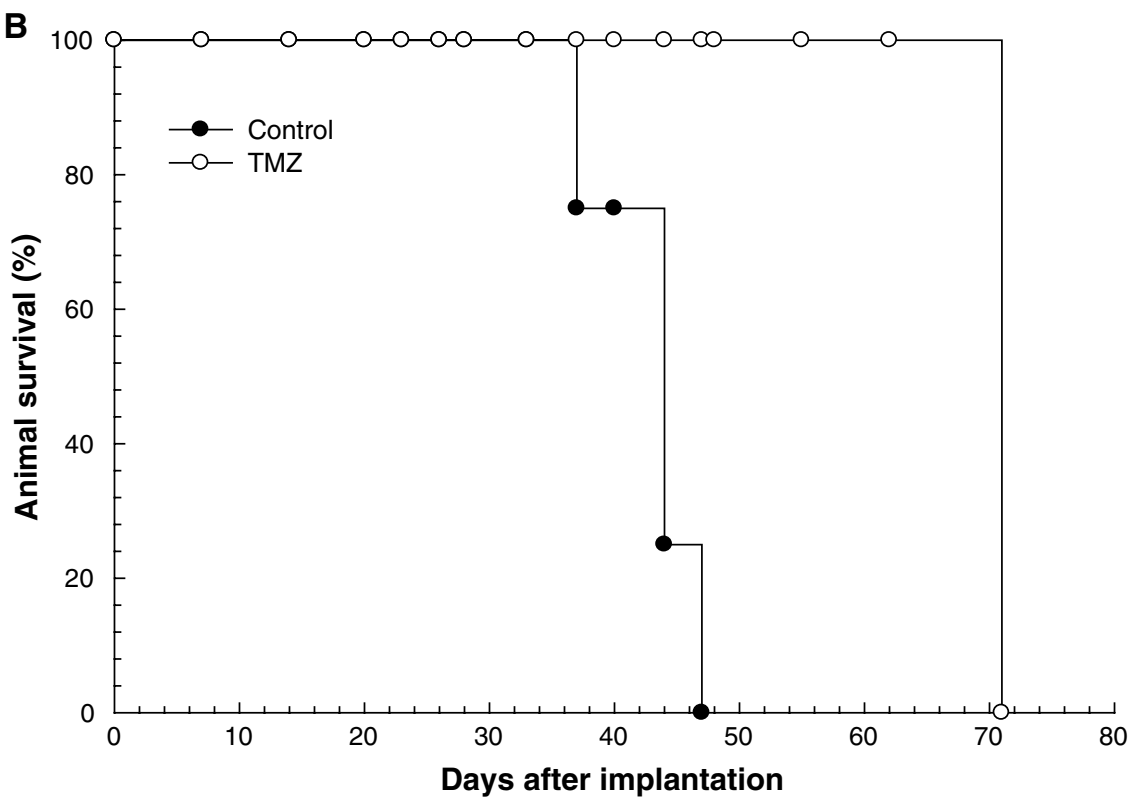


shown). All control animals with GS2 tumors required sacrificed by day 47 , with large pontine tumors present.

\section{Discussion}

In this study, we demonstrate the development of a xenograft model for brainstem glioma that has been adapted to facilitate pre-clinical testing of new therapies for the treatment of this cancer. The injection of luciferase-modified human glioblastoma cells in athymic rats produces brainstem tumors with a consistent rate of growth, and permits the use of BLI to quantitatively measure extent and duration of tumor response to therapy. For animal subjects receiving injection of tumor cells, histopathologic analysis revealed that tumor growth is primarily restricted to the pons, and as such, our approach results in an anatomically accurate modeling for this type of brain tumor.

Other brainstem tumor models using 9L and F98 rat glioma cell lines have been reported [20-24]. A potential limitation of rodent tumor-derived systems is that the response of rodent cell lines to treatment with anti-cancer agents can differ from that of human glioma cells [25-27]. Human glioma cell lines offer a distinct advantage with regard to this concern [7-9].

Among the five human GBM cell types used in the current study, we observed variable patterns of growth within the brainstem. U-87 MG and U-251 MG grew as well-defined tumors with sharply demarcated margins that do not recapitulate the observed pattern of pontine glioma growth in humans. Glioblastoma xenograft cell lines (GBM6, GBM14), and the glioblastoma neurosphere cell line GS2, all showed a greater extent of infiltration relative to U-87 MG and U-251 MG, but demonstrated lesser infiltration than has been observed following their supratentorial injection and growth in athymic mice (Fig. 1b). This may be due to the greater compaction of white matter pathways in the pons as compared to the cerebral hemispheres.

A refinement of our brainstem xenograft model is the use of BLI, which is a cost-effective, efficient, and accurate means for assessing tumor growth and response to therapy. In our time course study following injection of U-87 MG cells, bioluminescence signal was readily detectable on day 10 following tumor cell implantation, and well in advance of animals developing neurological symptoms. With respect to therapy response, the anti-tumor effect of TMZ treatment was readily detected by BLI, and the BLI effects of treatment foreshadowed corresponding increases in animal survival. These findings suggest that BLI can be used as an accurate surrogate to survival for assessing effect of therapy. One limitation of BLI is that metabolism of luciferin and photon emission can occur in non-dividing cells that are still viable. Therefore, loss of luminescent signal may not always correlate with tumor cell killing. It should also be noted that the sensitivity of U-87 MG and GS2 cells to TMZ is known and this drug was selected primarily to test the effectiveness of the model system rather than screen a potential therapeutic agent. In fact, clinical trials have not shown a meaningful response to TMZ in the treatment of pontine gliomas in children [28].

In the current era, most diffusely infiltrating brainstem gliomas are treated without a surgical biopsy [1]. The corresponding lack of tissue samples has resulted in a limited understanding of the underlying genetic and biochemical abnormalities associated with these tumors [29], and may explain why chemotherapeutic agents that have shown efficacy in other brain tumors have not been especially effective in clinical trials for brainstem gliomas. This limitation is of some concern for the model we present here, since all tumor cell sources we have used were obtained from adult GBM. A final limitation is that immunotherapy approaches cannot be effectively tested in immunodeficient animals. Nonetheless, this model is anatomically acccurate, allows ongoing quantitative assessment of tumor response, and can be easily adapted to establish a biologically faithful model if an adequate tissue specimen from a brainstem glioma becomes available in the future. In total, our approach, in which appropriate anatomic modeling is combined with BLI monitoring, should greatly enhance the testing of new therapeutics, as well as testing new strategies to deliver therapeutic agents, such as convection-enhanced delivery [2-4].

Acknowledgments This work was supported by the Pediatric Brain Tumor Foundation Pre-Institute Award, a Career Development Award (to AB) provided by the Brain Tumor SPORE at UCSF, Timmy's Rainbow Foundation for Brainstem Tumor Research, and National Institution of Health RO1 CA107268. NG is supported by K08 NS055061 (NIH/NINDS). The authors thank Lily J. Hu for technical assistance and Ilona Garner for editorial assistance.

Open Access This article is distributed under the terms of the Creative Commons Attribution Noncommercial License which permits any noncommercial use, distribution, and reproduction in any medium, provided the original author(s) and source are credited.

\section{References}

1. Recinos PF, Sciubba DM, Jallo GI (2007) Brainstem tumors: where are we today? Pediatr Neurosurg 43:192-201

2. Finlay JL, Zacharoulis S (2005) The treatment of high grade gliomas and diffuse intrinsic pontine tumors of childhood and adolescence: a historical- and futuristic-perspective. J Neurooncol 75:253-266

3. Avgeropoulos NG, Batchelor TT (1999) New treatment strategies for malignant gliomas. Oncologist 4:209-224

4. Huynh GH, Deen DF, Szoka FC Jr (2006) Barriers to carrier mediated drug and gene delivery to brain tumors. J Control Release 110:236-259 
5. Thorne RG, Pronk GJ, Padmanabhan V, Frey WHII (2004) Delivery of insulin-like growth factor-I to the rat brain and spinal cord along olfactory and trigeminal pathways following intranasal administration. Neuroscience 127:481-496

6. Hashizume R, Ozawa T, Gryaznov SM, Bollen AW, Lamborn KR, Frey WH 2nd, Deen DF (2008) New therapeutic approach for brain tumors: intranasal delivery of telomerase inhibitor GRN163. Neuro Oncol 10:112-120

7. Ozawa T, Wang J, Hu LJ, Bollen AW, Lamborn KR, Deen DF (2002) Growth of human glioblastomas as xenografts in the brains of athymic rats. In Vivo 16:55-60

8. Ozawa T, Gryaznov SM, Hu LJ, Pongracz K, Santos RA, Bollen AW, Lamborn KR, Deen DF (2004) Antitumor effects of specific telomerase inhibitor GRN163 in human glioblastoma xenografts. Neuro Oncol 6:218-226

9. Ozawa T, Santos RA, Lamborn KR, Bauer WF, Koo MS, Kahl SB, Deen DF (2004) In vivo evaluation of the boronated porphyrin TABP-1 in U-87 MG intracerebral human glioblastoma xenografts. Mol Pharm 1:368-374

10. Ozawa T, Faddegon BA, Hu LJ, Bollen AW, Lamborn KR, Deen DF (2006) Response of intracerebral human glioblastoma xenografts to multifraction radiation exposures. Int $\mathrm{J}$ Radiat Oncol Biol Phys 66:263-270

11. Edinger M, Cao YA, Hornig YS, Jenkins DE, Verneris MR, Bachmann MH, Negrin RS, Contag CH (2002) Advancing animal models of neoplasia through in vivo bioluminescence imaging. Eur J Cancer 38:2128-2136

12. Gross S, Piwnica-Worms D (2005) Spying on cancer: molecular imaging in vivo with genetically encoded reporters. Cancer Cell 7:5-15

13. Rehemtulla A, Stegman LD, Cardozo SJ, Gupta S, Hall DE, Contag CH, Ross BD (2000) Rapid and quantitative assessment of cancer treatment response using in vivo bioluminescence imaging. Neoplasia 2:491-495

14. Dinca E, Sarkaria J, Schroeder M, Carlson B, Voicu R, Berger M, James C (2007) Bioluminescence monitoring of intracranial glioblastoma xenograft response to primary and salvage temozolomide therapy. J Neurosurg 107:610-616

15. Kang SH, Cho HT, Devi S, Zhang Z, Escuin D, Liang Z, Mao H, Brat DJ, Olson JJ, Simons JW, Lavallee TM, Giannakakou P, Van Meir EG, Shim H (2006) Antitumor effect of 2-methoxyestradiol in a rat orthotopic brain tumor model. Cancer Res 66:11991-11997

16. Sarkaria JN, Yang L, Grogan PT, Kitange GJ, Carlson BL, Schroeder MA, Galanis E, Giannini C, Wu W, Dinca EB, James CD (2007) Identification of molecular characteristics correlated with glioblastoma sensitivity to EGFR kinase inhibition through use of an intracranial xenograft test panel. Mol Cancer Ther 6:1167-1174

17. Gunther HS, Schmidt NO, Phillips HS, Kemming D, Kharbanda S, Soriano R, Modrusan Z, Meissner H, Westphal M, Lamszus K (2008) Glioblastoma-derived stem cell-enriched cultures form distinct subgroups according to molecular and phenotypic criteria. Oncogene 27:2897-2909

18. Giannini C, Sarkaria JN, Saito A, Uhm JH, Galanis E, Carlson BL, Schroeder MA, James CD (2005) Patient tumor EGFR and PDGFRA gene amplifications retained in an invasive intracranial xenograft model of glioblastoma multiforme. Neuro Oncol 7:164-176

19. Hasegawa K, Pham L, O’Connor MK, Federspiel MJ, Russell SJ, Peng KW (2006) Dual therapy of ovarian cancer using measles viruses expressing carcinoembryonic antigen and sodium iodide symporter. Clin Cancer Res 12:1868-1875

20. Carson BS Sr, Wu Q, Tyler B, Sukay L, Raychaudhuri R, DiMeco F, Clatterbuck RE, Olivi A, Guarnieri M (2002) New approach to tumor therapy for inoperable areas of the brain: chronic intraparenchymal drug delivery. J Neurooncol 60:151158

21. Wu Q, Tyler B, Sukay L, Rhines L, DiMeco F, Clatterbuck RE, Guarnieri M, Carson BS Sr (2002) Experimental rodent models of brainstem tumors. Vet Pathol 39:293-299

22. Jallo GI, Penno M, Sukay L, Liu JY, Tyler B, Lee J, Carson BS, Guarnieri M (2005) Experimental models of brainstem tumors: development of a neonatal rat model. Childs Nerv Syst 21:399403

23. Lee J, Jallo GI, Guarnieri M, Carson BS Sr, Penno MB (2005) A novel brainstem tumor model: guide screw technology with functional, radiological, and histopathological characterization. Neurosurg Focus 18:E11

24. Jallo GI, Volkov A, Wong C, Carson BS Sr, Penno MB (2006) A novel brainstem tumor model: functional and histopathological characterization. Childs Nerv Syst 22:1519-1525

25. Tokuda K, Bodell WJ (1988) Cytotoxicity and induction of sister chromatid exchanges in human and rodent brain tumor cells treated with alkylating chemotherapeutic agents. Cancer Res 48:3100-3105

26. Oztopcu P, Kabadere S, Mercangoz A, Uyar R (2004) Comparison of vitamins $\mathrm{K} 1, \mathrm{~K} 2$ and $\mathrm{K} 3$ effects on growth of rat glioma and human glioblastoma multiforme cells in vitro. Acta Neurol Belg 104:106-110

27. Yaz G, Kabadere S, Oztopcu P, Durmaz R, Uyar R (2004) Comparison of the antiproliferative properties of antiestrogenic drugs (nafoxidine and clomiphene) on glioma cells in vitro. Am J Clin Oncol 27:384-388

28. Broniscer A, Iacono L, Chintagumpala M, Fouladi M, Wallace D, Bowers DC, Stewart C, Krasin MJ, Gajjar A (2005) Role of temozolomide after radiotherapy for newly diagnosed diffuse brainstem glioma in children: results of a multiinstitutional study (SJHG-98). Cancer 103:133-139

29. Gilbertson RJ, Hill DA, Hernan R, Kocak M, Geyer R, Olson J, Gajjar A, Rush L, Hamilton RL, Finkelstein SD, Pollack IF (2003) ERBB1 is amplified and overexpressed in high-grade diffusely infiltrative pediatric brain stem glioma. Clin Cancer Res 9:3620-3624 